\title{
Sobredosis de mirtazapina con evolución benigna
}

\author{
M. Raja y A. Azzoni \\ Servicio Psiquiátrico de Diagnóstico y Tratamiento, Hospital Espíritu Santo en Sassia, Roma, Italia
}

El antidepresivo mirtazapina (MTZ) se introdujo en el mercado por primera vez en los Países Bajos, en 1994. Hasta ahora, se ha dispuesto de pocos datos sobre el riesgo de sobredosis de MTZ, ya que ha habido únicamente cinco informes (MEDLINE, marzo de 2001) de intento de suicidio con este medicamento [1-5].

Comunicamos un caso de sobredosis de MTZ a causa de intención suicida, con recuperación completa y sin ninguna complicación.

Una mujer de 63 años afectada por trastorno bipolar, fase depresiva, fue admitida en un hospital general 3 horas después de la ingestión de más de 30 comprimidos de mirtazapina (Remeron ${ }^{\circledast}$ ) (30 mg). No había antecedentes de enfermedad médica actual o anterior importante. En los 30 días previos, la paciente había tomado mirtazapina $(15 \mathrm{mg} / \mathrm{d})$, citalopram $(20 \mathrm{mg} / \mathrm{d})$, risperidona $(2 \mathrm{mg} / \mathrm{d})$ y loracepam $(1 \mathrm{mg} / \mathrm{d})$. El padre y el hermano de la paciente se habían suicidado arrojándose al vacío. Dos hermanas y un hermano de la paciente sufrían trastorno del estado de ánimo. En la admisión, la paciente estaba alerta y aparentemente orientada. Los signos vitales, el ECG y la exploración física fueron normales. Se administró jarabe de ipecacuana para inducir vómitos. Ya que los signos clínicos de la paciente se mantenían estables, se la transfirió a una unidad de cuidados intensivos psiquiátricos. En los días siguientes, los signos vitales, el ECG (6), las pruebas de laboratorio y la exploración física fueron normales. El peso corporal era $60 \mathrm{~kg}$. Cuatro días después de la sobredosis de mirtazapina, se trató a la paciente con litio (hasta $300 \mathrm{mg}$, tres veces al día), valproato (hasta 500, dos veces al día) y fluracepam ( $30 \mathrm{mg}$, al acostarse) con mejoría moderada. La MTZ se absorbe bien después de la administración oral, llegando a concentraciones pico en plasma en un plazo de 2 horas. Se logran concentraciones de equili- brio en plasma en un plazo de 3-5 días. La MTZ se metaboliza completamente después de administración oral. Las vías principales de biotransformación son la desmetilación y la hidroxilación seguidas por conjugación de glucurónido. La semivida media de eliminación de la MTZ después de la administración oral es de 2040 horas (semivida media de 37 horas para las mujeres). Alrededor del $100 \%$ de la dosis oral se excreta vía orina y heces en un plazo de 4 días. La paciente había tomado al menos 30 veces una dosis diaria normal de MTZ. Fue admitida 3 horas después de la sobredosis. Así, es probable que la concentración pico en plasma se hubiera producido ya. Sin embargo, el estado clínico de la paciente se mantuvo estable, sin daño o complicaciones. Presentaba sólo sedación moderada sin ninguna anomalía en los signos vitales, el ECG, las pruebas de laboratorio o los síntomas clínicos. De acuerdo con informes anteriores, la MTZ parece ser un medicamento seguro en sobredosis, al menos cuando no hay implicación de múltiples fármacos. Sin embargo, se necesita vigilancia sistemática a largo plazo antes de sacar una conclusión firme sobre esta cuestión.

\section{BIBLIOGRAFÍA}

1 Bremner JD, Wingard P. Walshe TA. Safety of mirtazapine in overdose. J Clin Psychiatry 1998; 59: 233-5.

2 Gerritsen AW. Safety in overdose of mirtazapine: a case report. J Clin Psychiatry 1997; 58: 271.

3 Hoes MJ, Zeijpveld JH. First report of mirtazapine overdose. Int Clin Psychopharmacol 1996; 11: 247.

4 Holzbach R, Jahn H, Pajonk FG, Mahne C. Suicide attempts with mirtazapine overdose without complications. Biol Psychiatry 1998, 44: 925-6.

5 Retz W, Maier S, Maris F, Rosler M. Non fatal mirtazapine overdose. Int Clin Psychopharmacol 1998; 13: 277-9. 